\title{
Gobierno corporativo e insider trading. La concentración de la propiedad como variable moderadora del rol de control de los consejos*
}

Corporate management and insider trading. The concentration of property as a moderating variable for controlling roles on boards

Governança corporativa e insider trading. A concentração de propriedade como uma variável moderadora do papel de controle dos conselhos

José E. Tobar Arias ${ }^{\text {a }}$

Universidad Icesi, Colombia

jetobar@icesi.edu.co

ORCID: https://orcid.org/0000-0002-0462-3569

DOI: https://doi.org/10.11144/Javeriana.cao34.gcitcp

Andres Mora Valencia

Universidad de los Andes, Colombia

Recibido: 19 Enero 2021

ORCID: https://orcid.org/0000-0001-9381-8319

Aceptado: 08 Octubre 2021

\author{
Esther B. del Brio González \\ Universidad de Salamanca, España \\ ORCID: https://orcid.org/0000-0001-5990-7377
}

\section{Resumen:}

El propósito de este documento es doble: en primer lugar, se realiza un análisis del efecto directo de la estructura de propiedad y de los consejos de administración sobre el insider trading. En segundo lugar, examinamos el posible efecto moderador que tiene la concentración de la propiedad sobre el papel disciplinario de los consejos a la hora de prevenir esta práctica de mercado. Los resultados de la regresión múltiple y la regresión múltiple jerárquica muestran un efecto negativo tanto de la concentración de la propiedad como de la porción de directores independientes sobre el insider trading. En cuanto al efecto moderador, se evidencia que el rol de control de los consejos está influenciado por la concentración de propiedad.

Códigos JEL: G30, G14.

Palabras clave: Gobierno corporativo, insider trading, estructura de propiedad.

\section{Abstract:}

The purpose of this paper is twofold: First, an analysis of the direct effect of ownership structure and boards of directors on insider trading. Secondly, we examine the possible moderating effect the concentration of ownership has on the disciplinary role of boards in preventing this market practice. The results of the multiple regression and hierarchical multiple regression show the negative effect of both concentration of property and the portion of independent directors on insider trading. Regarding the moderating effect, there is evidence that the controlling roles on the boards are influenced by the concentration of property.

JEL Codes: G30, G14.

Keywords: Corporate governance, insider trading, ownership structure.

\section{Resumo:}

O objetivo deste documento é duplo: em primeiro lugar, é realizada uma análise do efeito direto da estrutura de propriedade e conselhos de administração sobre o insider trading. Em segundo lugar, examinamos o possível efeito moderador que a concentração de propriedade tem sobre o papel disciplinar dos conselhos no momento da prevenção dessa prática de mercado. Os resultados da regressão múltipla e da regressão múltipla hierárquica mostram um efeito negativo tanto da concentração de propriedade quanto da parcela de conselheiros independentes no insider trading. No que se refere ao efeito moderador, fica evidente que o papel de controle dos conselhos é influenciado pela concentração da propriedade.

Códigos JEL: G30, G14.

Palavras-chave: Governança corporativa, insider trading, estrutura de propriedade.

Notas de autor

\footnotetext{
${ }^{a}$ Autor de correspondencia. Correo electrónico: jetobar@icesi.edu.co
} 


\section{Introducción}

La transparencia en los mercados de valores es fundamental para una asignación de recursos de capital eficiente, en este sentido, los agentes económicos deben disponer de información real, relevante y oportuna para garantizar una acertada toma de decisiones y una adecuada formación de precios de los títulos que se negocian. Sin embargo, este proceso puede verse afectado por las imperfecciones del mercado causadas por conflictos de interés y asimetrías de información entre directivos y propietarios (Jensen \& Meckling, 1979) o entre insiders y demás agentes del mercado que afectan la eficiencia y dejan margen a comportamientos oportunistas del agente con mejor información, en detrimento de la parte menos informada (Fishman \& Hagerty, 1992; Georgakopoulos, 1993; Goshen \& Parchomovsky, 2001). La magnitud de estas imperfecciones y su efecto en la formación de precios depende, en parte, de los mecanismos de control internos y externos de cada mercado.

Una de las imperfecciones del mercado la constituye el insider trading, un comportamiento derivado de las asimetrías de información que permiten a un grupo de personas -insiders-, quienes tienen una relación laboral o funcional con la empresa, acceder a información privilegiada y usarla posteriormente en las operaciones de mercado abierto, obteniendo rentabilidades superiores a la media del mercado. Esta práctica afecta la eficiencia y el desarrollo de los mercados (Beck \& Levine, 2002; Demirgüç-Kunt \& Maksimovic, 1998, 2002; Rajan \& Zingales, 1996). El insider trading es considerado por la mayoría de los gobiernos como una práctica perjudicial para la integridad de sus mercados de capitales ${ }^{1}$. En este sentido, los órganos de control de la mayoría de los mercados han dirigido sus esfuerzos a prevenir, investigar y sancionar el uso indebido de la información privilegiada (insider trading), con la finalidad de garantizar un trato justo y equitativo a los inversores y evitar el deterioro de la confianza en los mercados.

El nivel de interés de los gobiernos por aplicar estas regulaciones se evidencia en el trabajo desarrollado por Bhattacharya \& Daouk (2002); estos autores encuentran que para el año 1998, los 22 países considerados desarrollados contaban con regulación de insider trading, y cuatro de cada cinco de los 81 países considerados emergentes, también la tenían.

A pesar del mayor grado de desarrollo y aceptación que ha mostrado la regulación del insider trading a través de los años, la evidencia empírica obtenida en numerosos países sugiere que los directivos empresariales mantienen su capacidad de obtener rentabilidades superiores a la media del mercado (Aboody \& Lev, 2000; Lakonishok \& Lee, 2001; Hillier \& Marshall, 2002; Friederich, Gregory, Matatko et al., 2002; Jeng, Metrick \& Zeckhauser, 2003; Del Brío, Miguel \& Perote, 2002; Fidrmuc, Goergen \& Renneboog, 2006; Jagolinzer, 2009; Betzer \& Theissen, 2009; Del Brío, De Miguel \& Tobar, 2010), lo cual contraviene la hipótesis fuerte de eficiencia de los mercados y pone en entredicho la eficacia de la regulación (Bhattacharya \& Daouk, 2002; Bris, 2005) y de la validez de otros mecanismos externos de gobierno corporativo a la hora de reducir la asimetría en la información entre insiders y outsiders.

Los mercados competitivos de bienes y servicios, el mercado de capitales y el mercado de control corporativo proporcionan mecanismos disciplinarios que ayudan a alinear los objetivos e intereses entre principal y agente. Sin embargo, estos mecanismos de control externo son mucho más eficientes en países de tradición jurídica del Common - Law en comparación con países de tradición jurídica Civil - Law. (La Porta, Lopez-De-Silanes, Shleifer et al., 1997; La Porta, Lopez-de-Silanes \& Shleifer, 1999); situación que se intensifica en aquellos entornos caracterizados por una débil protección al inversor (La Porta, Lopez-deSilanes, Shleifer et al., 1998).

Ante los grandes escándalos corporativos acaecidos en las últimas dos décadas se ha intensificado el interés de reguladores y académicos por analizar la eficacia de los sistemas internos de gobierno corporativo de las empresas. Desde la perspectiva de la teoría de la agencia, el mayor número de estudios se ha centrado en analizar la eficacia de estos sistemas internos de gobierno en la prevención de comportamientos oportunistas 
de la gerencia que vayan en contra de los intereses de los accionistas (Fama, 1980; Fama \& Jensen, 1983; Jensen, 1993). Por lo general, estos estudios parten del supuesto de que los mecanismos internos de control operan independientemente, desconociendo, la interacción entre ellos y los posibles efectos mediadores y moderadores que pueden afectar la eficacia de las diferentes prácticas de la gobernanza empresarial.

Este trabajo pretende llenar este vacío de la literatura, para lo cual se plantean dos objetivos: (i) hacer un análisis del efecto directo que tienen sobre el insider trading la concentración de la propiedad y la independencia del consejo de administración; y (ii) evaluar el posible efecto moderador que tiene la concentración de la propiedad sobre el rol disciplinante de los consejos a la hora de prevenir esta práctica de mercado.

Este trabajo parte del supuesto que el gobierno corporativo afecta al insider trading y no viceversa, relación causal que ha sido soportada conceptual y empíricamente ${ }^{2}$. La literatura financiera clásica ha establecido que un buen gobierno corporativo mitiga los costos de agencia asociados con la separación entre propiedad y control (Shleifer \& Vishny, 1997; Bushman \& Smith, 2001). De igual manera, la teoría de la agencia ha establecido que en escenarios de asimetría informativa los insiders pueden ejercer su propio interés a costa de otros accionistas (Jensen \& Meckling, 1979). Así, el concepto de gobierno corporativo abarca una serie de mecanismos que la literatura ha identificado como eficaces a la hora de disminuir dichas asimetrías y asegurar que los directivos actúen en línea con los intereses de los accionistas y no en beneficio propio. Vale resaltar la idea de que en escenarios con estructuras de propiedad relativamente estables en el tiempo, disminuye la posibilidad de causalidad inversa (La Porta, Lopez-de-Silanes, \& Shleifer, 1999; Zhou, 2001).

Desde el punto de vista empírico, Rozanov (2008) analizó un subconjunto de empresas en las que su CEO fue reemplazado recientemente (escenario donde es menos probable que un nuevo director ejecutivo tenga un impacto significativo en la composición de los consejos y en la estructura de propiedad), sus resultados sugieren que en empresas con consejos mayoritariamente independientes y con una mayor concentración de propiedad los insiders tienen menos posibilidades de realizar prácticas de insider trading.

En virtud de lo anterior es muy plausible esperar que el gobierno corporativo disminuya las posibilidades para que los insiders exploten su ventaja informativa, y debilita la idea que los insiders puedan tener la capacidad para perfilar el tipo de consejo y la estructura de propiedad a fines a sus intereses.

Para lograr los objetivos planteados se tomaron como muestra las operaciones de compra de los insiders españoles durante el periodo 1999 - 2007. La razón de concentrarnos solo en las operaciones de compra se sustenta en el mayor contenido informativo de estas operaciones, soportado en abundante evidencia empírica (Friederich et al., 2002; Hillier \& Marshall, 2002; Jeng et al., 2003; Fidrmuc et al., 2006; Del Brío, De Miguel \& Tobar, 2010; Qiu, He \& Xiao, 2018).

Se procedió a calcular sus rentabilidades anormales de las operaciones de compra mediante la aplicación de la metodología de estudio de eventos, donde la variable a explicar son las rentabilidades anormales acumuladas durante el día previo y posterior a la fecha de las operaciones de compra de los insiders $C A R(-3,+3)$, considerada en la literatura financiera como una variable proxy adecuada del nivel del insider trading ${ }^{3}$, puesto que se considera que la rentabilidad de las operaciones de los insiders refleja la materialidad de su ventaja informativa (Rogoff, 1964).

Los resultados obtenidos soportan una relación negativa y significativa entre la concentración de la propiedad y las rentabilidades anormales de las operaciones de compra, corroborando los beneficios de la presencia de un gran accionista a la hora de prevenir comportamientos oportunistas de los insiders.

En cuanto a las variables del consejo, los resultados indican una relación negativa y significativa entre la fracción de consejeros externos y las rentabilidades de las operaciones de compra de los insiders. Sin embargo, al dividir los consejeros externos entre independientes y dominicales ${ }^{4}$, los resultados se mantienen sólo para los independientes, lo que sugiere una mayor capacidad de control sobre el insider trading de los consejeros independientes en relación con el control ejercido por los consejeros dominicales. Este hallazgo concuerda con las recomendaciones de los códigos de buen gobierno de la mayoría de países 5 . 
$\mathrm{Al}$ analizar el efecto conjunto de la concentración de la propiedad y de los consejos de administración los resultados indican que la concentración de propiedad tiene un efecto moderador en el control que realizan los consejos sobre el insider trading. La evidencia muestra que en empresas con mayor concentración de propiedad, el rol de control de los consejos sobre el insider trading es mucho menor (mayores rentabilidades anormales), situación que se hace más crítica cuando se analiza el control ejercido por consejeros dominicales. Esto respalda la idea de que los intereses de los consejeros dominicales pueden estar más alineados con los intereses del gran accionista a diferencia de los consejeros independientes.

Estos resultados ponen de relieve la importancia de tener en cuenta la interacción entre los diferentes elementos de gobierno corporativo y sus posibles efectos mediadores o moderadores de unos elementos, entre unos y otros, que pueden afectar la eficacia de las diferentes prácticas de la gobernanza empresarial.

\section{Literatura previa e hipótesis}

A continuación se hace una revisión de los trabajos más relevantes que analizan la eficacia de la concentración de propiedad y la independencia del consejo a la hora de prevenir el uso de información privilegiada.

\section{Concentración de propiedad e insider trading}

El conflicto de intereses generado por la separación entre propiedad y control (Berle \& Means, 1932), formalizado por la teoría de la agencia (Fama \& Jensen, 1983; Jensen \& Meckling, 1979), sugieren que los accionistas desean maximizar el valor de la empresa, mientras que los gerentes actúan en su propio interés, que no necesariamente coincide con los intereses de los accionistas.

Desde un enfoque de control de la concentración de la propiedad, la literatura financiera sugiere que el gran accionista cuenta con los incentivos necesarios para controlar a los insiders, debido a que parcialmente pueden internalizar los beneficios de su esfuerzo de control (Grossman \& Hart, 1986). Se esperaría entonces que el mayor control ejercido a la actividad directiva disminuya los problemas de asimetrías informativas y mejore el desempeño empresarial (Agrawal \& Mandelker, 1990; Alonso-Bonis \& de Andrés-Alonso, 2007; Claessens, Djankov, Fan et al., 2002; Jensen, 1989; Maug, 1998; Morck, Shleifer, \& Vishny, 1988; Shleifer \& Vishny, 1986, 1997).

Fidrmuc et al. (2006) analizaron la relación entre concentración de propiedad e insider trading desde la óptica del gobierno corporativo, y encontraron que en grandes empresas del Reino Unido el control por grandes accionistas ${ }^{6}$ externos reduce las rentabilidades del insider trading. Para España, Del Brío \& Perote (2007) soportan una relación positiva entre propiedad difusa y rentabilidades de las operaciones de insiders. Rozanov (2008) para Estados Unidos, identifica una relación negativa entre concentración de la propiedad (como proxyde buen gobierno) y el insider trading. Cziraki, De Goeij \& Renneboog (2014) para Holanda, encuentran una relación positiva entre el insider trading y la presencia de blockholders; estos autores justifican estos resultados al considerar que en empresas con un buen gobierno corporativo los insiders tienen menos posibilidades de obtener beneficios privados del control y, por tanto, optan por explotar su ventaja informativa a través del insider trading. He \& Rui (2016) para China, encuentran que el retorno anormal de las operaciones compra de los insiders es una función convexa al porcentaje de propiedad del principal accionista.

Por último, la investigación de Tobar, Del Brío \& De Miguel (2017) para el mercado español evidenció la coexistencia de los efectos de control y de expropiación entre concentración de propiedad y los CAR's de las operaciones de compra de los insiders. Sin embargo, al derivar el correspondiente punto de corte de la relación se determina que solo a partir de una muy alta concentración de propiedad del principal accionista (mayor al 55\%) se da un efecto de atrincheramiento (positivo sobre el insider trading). En este trabajo solo 
nos interesa analizar el efecto dominante de control de la concentración de propiedad, por lo tanto, se plantea la siguiente hipótesis:

Hipótesis 1. La concentración de la propiedad ejerce un efecto negativo sobre el insider trading.

\section{Independencia del Consejo y el insider trading}

En lo que respecta a la independencia del consejo, esta característica tal vez sea la que ha generado mayor debate en los últimos años como consecuencia de los grandes fraudes corporativos asociados a comportamientos oportunistas de los insiders. En este sentido, y a pesar de que algunos trabajos no soportan la idea generalizada de las bondades de un consejo mayoritariamente independiente (Yermack, 1996; Agrawal \& Knoeber, 1996; Romano, 1998; Klein, 1998; Bhagat \& Black, 2002; Park \& Shin, 2004; De Jong, DeJong, Mertens \& Wasley, 2005; Howton, 2006; Lin, Ma \& Su, 2009), las presiones legales e institucionales han hecho que prevalezca la función de control del consejo, las cuales se hacen evidentes al observar las nuevas exigencias impuestas tanto por la ley Sarbanes Oxley Act 2002 para Estados Unidos, como por la regulación de importantes bolsas de valores como la NYSE y el NASDAQ (Dah, Frye \& Hurst, 2014). Esta visión negativa de los consejeros con un rol ejecutivo se sustenta en la idea de la débil función de control que se les atribuye dentro del consejo debido a su tendencia de actuar en su propio interés, por lo que deben ser controlados para evitar comportamientos oportunistas (Fama \& Jensen, 1983; Shleifer \& Vishny, 1997).

En cuanto a la relación entre consejeros independientes e insider trading se encuentran trabajos como los de Rozanov (2008) que para una muestra de empresas de los Estados Unidos correspondiente al periodo 1996-2006 identifican una relación negativa entre el porcentaje de consejeros externos y el insider trading. Ravina \& Sapienza (2009) analizó operaciones de insiders de empresas de los Estados Unidos para el periodo 1986-2003 u encontró que los consejeros independientes ganan importantes rentabilidades anormales en operaciones de compra de su empresa, y que la diferencia con las rentabilidades obtenidas por los ejecutivos de la empresa es relativamente pequeña, situación que se da especialmente en empresas con un gobierno corporativo deficiente.

Para el mercado español, la Comisión Nacional del Mercado de Valores (CNMV) ${ }^{7}$ clasificó a los consejeros en tres categorías: ejecutivos, independientes y dominicales. La figura de consejeros dominicales es un tipo especial de consejero externo que incluye a aquellos que poseen una participación accionarial por encima o igual a la del umbral establecido legalmente. En la literatura financiera al conjunto de consejeros independientes y dominicales se les denomina consejeros externos, a los cuales se les presupone entre otras, una función de control sobre los insiders. Así, se plantea la hipótesis 2.

Hipótesis 2. Existe una relación negativa entre la fracción de consejeros externos y el insider trading.

En la hipótesis 2 se parte del supuesto de que tanto los consejeros independientes como los consejeros dominicales tienen los mismos incentivos para controlar comportamientos oportunistas de los insiders, pero la condición de accionista de los consejeros dominicales podría ser una restricción a su capacidad de control, tal y como lo sugiere la $\mathrm{CNMV}^{8}$. Por tanto, tratar a los dos tipos de consejeros como un solo grupo podría enmascarar el verdadero efecto que ejerce cada grupo sobre el insider trading. Esto lleva a plantear la hipótesis 3.

Hipótesis 3. Existe mayor control sobre el insider trading por parte de los consejeros independientes, en comparación con el control ejercido por los consejeros dominicales. 


\section{Efecto moderador de la concentración de la propiedad sobre la relación entre consejo e insider trading}

La literatura financiera sugiere que las características del consejo de administración dependen en gran medida del tipo de estructura de propiedad de la empresa, que indirectamente afecta su eficacia supervisora a la hora de controlar comportamientos oportunistas de los insiders (Bathala \& Rao, 1995; Donnelly \& Kelly, 2005).

La literatura soporta la idea de que los grandes accionistas cuentan con el incentivo y poder necesario para disciplinar directamente a los insiders, por ello, tendrán una menor dependencia de las funciones de control del consejo (Hermalin \& Weisbach, 1988; Méndez \& García, 2007). Otros trabajos empíricos han probado una relación negativa entre la fracción de consejeros externos y el nivel de concentración de la propiedad a nivel empresa y país, sugiriendo que los grandes accionistas tienen mayor preferencia por consejeros ejecutivos, quienes estarán más alineados con sus propios intereses, que no necesariamente deberán coincidir con los intereses de los pequeños accionistas (Kim, Kitsabunnarat-Chatjuthamard, \& Nofsinger, 2007).

Las hipótesis planteadas hasta acá sugieren que tanto la concentración de la propiedad como la independencia del consejo limitan las prácticas de insider trading. Sin embargo, en este apartado se busca determinar si el rol de control del consejo sobre el insider trading se ve moderado por el nivel de concentración de propiedad, es decir, se quiere evidenciar si la capacidad de control del consejo varía ante diferentes niveles de concentración de propiedad.

Se analiza el efecto de la estructura de propiedad sobre el consejo y no viceversa, ya que se considera que el tipo de accionista y su participación en la propiedad pueden generar los incentivos necesarios para perfilar consejos más afines a sus intereses, que no necesariamente deben estar alineados con los de otros grupos de interés. La presencia de una interacción estadísticamente significativa indicaría que los dos mecanismos de control no son independientes entre sí. En este sentido, se plantea la hipótesis 4.

Hipótesis 4. La relación entre la fracción de consejeros externos y el insider trading es moderada por el nivel de concentración de propiedad.

De igual forma, se busca probar si el posible efecto moderador de la concentración de la propiedad sobre el control ejercido por los consejeros difiere dependiendo del tipo de consejero (independientes o dominicales). De acuerdo con lo anterior, se plantea la hipótesis 5.

Hipótesis 5. El efecto moderador de la concentración de la propiedad sobre el rol de control de los consejeros es mayor para los consejeros dominicales que para los consejeros independientes.

\section{Muestra, variables y metodología}

\section{Muestra}

La CNMV para España requiere a los insiders de las empresas cotizadas que informen sobre las operaciones realizadas con valores de su propia empresa, a partir de estas comunicaciones, la CNMV alimenta una base de datos, la cual fue empleada en este trabajo, utilizando las operaciones de compra del periodo 2000-2007. El análisis se centra en operaciones de compra de empresas no financieras debido al mayor contenido informativo de estas operaciones, soportado un gran número de trabajos (Del Brío, De Miguel, \& Tobar, 2010; Fidrmuc et al., 2006; Korczak, Korczak, \& Lasfer, 2010). Se tomó el 2007 como el último año de la muestra debido a que el mercado de capitales inició su declive derivado de la crisis financiera global. Después de aplicar los filtros requeridos en la metodología de estudio de eventos, la base de datos final está compuesta por un total de 2.233 operaciones de compra correspondientes en promedio a 69 empresas no financieras (ver tabla 1). En 
la tabla 1 se observa cierta estabilidad en la ratio de operaciones por empresa, siendo el año 2002 el periodo de menor volumen (ratio $=3,0$ ), y el periodo 2007 el de mayor volumen (ratio $=4,5$ ).

Las variables relacionadas con la estructura de propiedad fueron obtenidas de la base de datos internacional Amadeus, las características del consejo fueron obtenidas para cada año del Índice Spencer Stuart de Consejos de Administración, y el resto de las variables financieras se obtuvieron de la base de datos internacional Datastream/Worldscope.

TABLA 1

Distribución cronológica de la muestra

\begin{tabular}{lccccccccc}
\hline & $\mathbf{2 0 0 0}$ & $\mathbf{2 0 0 1}$ & $\mathbf{2 0 0 2}$ & $\mathbf{2 0 0 3}$ & $\mathbf{2 0 0 4}$ & $\mathbf{2 0 0 5}$ & $\mathbf{2 0 0 6}$ & $\mathbf{2 0 0 7}$ & Total \\
\hline Transacciones & 230 & 230 & 169 & 239 & 288 & 333 & 351 & 393 & 2.233 \\
\hline Empresas & 64 & 57 & 56 & 63 & 65 & 76 & 81 & 87 & 549 \\
\hline $\begin{array}{l}\text { Transacciones/ } \\
\text { Empresas }\end{array}$ & 3,6 & 4,0 & 3,0 & 3,8 & 4,4 & 4,4 & 4,3 & 4,5 & 3,9 \\
\hline
\end{tabular}

Fuente: elaboración propia con base de datos de la CNMV.

\section{Variables y metodología}

En la tabla 2 se exponen las variables utilizadas en el análisis. En la metodología se usa el estudio de eventos para el cálculo de las rentabilidades anormales, donde el evento de interés es cada operación individual realizada por los insiders. Para analizar los efectos directos de la concentración de la propiedad y de las características de interés del consejo sobre el insider trading se utilizaron modelos de regresión múltiple, estimados por la metodología de mínimos cuadrados ordinarios (MCO), y para analizar el posible efecto moderador de la estructura de propiedad se recurrió a un modelo de regresión múltiple jerárquica, en línea con lo planteado por Aiken, West, \& Reno, (1991), que prueban el efecto moderador cuando la interacción entre la variable predictora (independencia del consejo) y la variable moderadora (concentración de la propiedad) da lugar a un coeficiente de regresión significativo, y dicho coeficiente se relaciona con un incremento significativo en la varianza explicada. 
TABLA 2

Explicación de las variables dependientes e independientes

\begin{tabular}{|c|c|}
\hline \multicolumn{2}{|r|}{ Panel A. Variable dependiente } \\
\hline Variable & Descripción \\
\hline $\operatorname{CAR}(-3,+3)$ & $\begin{array}{l}\text { Rentabilidades anormales medios acumulados de las operaciones de compra } \\
\text { de los insiders, corresponde a las rentabilidades anormales acumuladas el día } \\
\text { del evento y los días anterior y posterior a este. }\end{array}$ \\
\hline \multicolumn{2}{|r|}{ Panel B. Variables explicativas y de control } \\
\hline \multicolumn{2}{|c|}{ Variables explicativas } \\
\hline SH & Porcentaje de propiedad retenida por el principal accionista \\
\hline EXT & $\begin{array}{l}\text { Fracción de consejeros externos en relación con el número total de } \\
\text { consejeros }\end{array}$ \\
\hline INDEP & $\begin{array}{l}\text { Fracción de consejeros independientes en relación con el número total de } \\
\text { consejeros }\end{array}$ \\
\hline DOMINI & $\begin{array}{l}\text { Fracción de consejeros dominicales en relación con el número total de } \\
\text { consejeros }\end{array}$ \\
\hline \multicolumn{2}{|c|}{ Variables de control } \\
\hline CONS & $\begin{array}{l}\text { Variable dummy que identifica cuando la operación de compra es realizada } \\
\text { por un consejcro }\end{array}$ \\
\hline$B S Z$ & $\begin{array}{l}\text { Tamaño del consejo medido como el logaritmo natural del número total de } \\
\text { consejeros }\end{array}$ \\
\hline$B_{-} L S Z$ & $\begin{array}{l}\text { Variable dummy que identifica a consejos considerados pequeños (aquellos } \\
\text { que están por debajo del valor medio en una desviación estándar) }\end{array}$ \\
\hline$B \_B S Z$ & $\begin{array}{l}\text { Variable dummy que identifica a consejos considerados grandes (aquellos } \\
\text { que superan al valor medio en una desviación estándar) }\end{array}$ \\
\hline$L V M K$ & $\begin{array}{l}\text { Tamaño de la empresa medido como el logaritmo natural de valor de } \\
\text { mercado de la empresa durante el año que se realiza la operación }\end{array}$ \\
\hline$L E V$ & $\begin{array}{l}\text { Nivel de endeudamiento de la empresa (leverage) medida como la ratio entro } \\
\text { total deuda de la empresa y el total de sus activos }\end{array}$ \\
\hline$B M$ & $\begin{array}{l}\text { Book-to-market medido como la ratio entre el valor contable de la empresa y } \\
\text { el valor de mercado }\end{array}$ \\
\hline OPSZ & $\begin{array}{l}\text { Tamaño de la operación de compra medido como la ratio entre el número de } \\
\text { acciones de la operación y el número de acciones en circulación de la } \\
\text { empresa en el año en que se realizó la operación }\end{array}$ \\
\hline$O P M$ & $\begin{array}{l}\text { Variable dummy que identifica cuando el insider ha realizado más de una } \\
\text { operación en la misma fecha }\end{array}$ \\
\hline
\end{tabular}

Fuente: elaboración propia.

\section{Resultados}

Antes de presentar los resultados de las regresiones, en la tabla 3 se muestran los estadísticos descriptivos de las principales variables. Como se puede apreciar, en promedio hay mayor fracción de consejeros externos $(80,5 \%)$ en relación con el número total de consejeros, seguido de la fracción de consejeros dominicales $(42,9 \%)$ y de consejeros independientes $(36 \%)$ en la muestra analizada.

TABLA 3

Datos estadísticos de las principales variables

\begin{tabular}{lrrrr}
\hline \multicolumn{1}{c}{ Variables } & \multicolumn{1}{c}{ Media } & \multicolumn{1}{c}{ Mínimo } & Máximo & $\begin{array}{c}\text { Desviación } \\
\text { estándar }\end{array}$ \\
\hline SH & 0,272 & 0,0010 & 0,8538 & 0,1803 \\
EXT & 0,805 & 0,1333 & 1 & 0,1081 \\
INDEPEN & 0,360 & 0 & 0,8667 & 0,1726 \\
DOMINI & 0,429 & 0 & 1 & 0,2030 \\
BSZ & 2,514 & 1,3863 & 3,1781 & 0,3094 \\
LVMK & 6,977 & 3,9354 & 9,9567 & 1,5289 \\
LEV & 0,308 & 0,0001 & 2,0575 & 0,2027 \\
BM & 0,507 & $-1,3219$ & 2,9636 & 0,4198 \\
OPSZ & 0,016 & 0,0001 & 0,8621 & 0,0684 \\
Total consejeros & 13 & 4 & 24 & 4,0197 \\
Consejeros externos & 10 & 2 & 20 & 3,7250 \\
Consejeros dominicales & 6 & 0 & 18 & 3,5592 \\
Consejeros independientes & 5 & 0 & 13 & 2,3977 \\
\hline
\end{tabular}

Fuente: elaboración propia. 


\section{Efecto directo de la concentración de propiedad sobre el insider trading}

Todos los modelos estimados (tablas 4 a 7 ) los coeficientes de la variable $(\mathrm{SH})_{i t}$ son negativos y significativos, lo que soporta una relación inversa entre el nivel de concentración de la propiedad y las rentabilidades anormales de los insiders, resultados que sugieren la idea de que la presencia de grandes accionistas mejora el control de la actividad directiva, y en este caso, restringe la posibilidad de que los insiders exploten su ventaja informativa en sus operaciones de compra. Los resultados son consistentes con los postulados de la teoría de la agencia: que los grandes accionistas tienen mayor incentivo para desempeñar un papel activo en las decisiones de la empresa porque ellos internalizan parcialmente los beneficios de su control (Grossman \& Hart, 1986).

TABLA 4

Efecto directo de la concentración de la propiedad y el insider trading

\begin{tabular}{|c|c|c|}
\hline Variables & Coeff. & t-stat. \\
\hline Constante & $-0,0128$ & $-1,557$ \\
\hline$S H$ & $-0,0237^{* * *}$ & $-3,505$ \\
\hline $\mathrm{CONS}^{1 /}$ & $-0,0016$ & $-0,569$ \\
\hline$L V M K$ & $0,0033^{* * * *}$ & 3,368 \\
\hline LEV & $-0,0175^{* * * *}$ & $-2,666$ \\
\hline$B M$ & 0,0003 & 0,063 \\
\hline OPSZ & $0,0387^{* * *}$ & 2,623 \\
\hline$O P M^{2}$ & 0,0042 & 1,476 \\
\hline$R^{2}$ ajustado & \multicolumn{2}{|c|}{2,8} \\
\hline$F$ & \multicolumn{2}{|c|}{5,101} \\
\hline$N$ & \multicolumn{2}{|c|}{1.016} \\
\hline \multicolumn{3}{|c|}{$\begin{array}{l}\text { "CONS: variable dummy que toma el valor de } 1 \text { cuando el insider que realizó la operación es un } \\
\text { consejero, y cero en otro caso. } \\
{ }^{2} \text { OPM: variable dummy que toma el valor de } 1 \text { cuando el insider realizó más de una compra en la } \\
\text { misma empresa y en la misma fecha. }\end{array}$} \\
\hline
\end{tabular}

\section{Efecto directo de los consejos de administración sobre el insider trading}

En cuanto a la composición del consejo, en el panel A y B de la tabla 5 se obtienen coeficientes negativos y significativos de la variable (EXT), resultados que inicialmente soportan el buen control por parte de los consejeros externos sobre el insider trading, respaldando la hipótesis 2. No obstante, al dividir los consejeros externos entre consejeros independientes y dominicales se observa en la tabla 6 (panel A y B) que los coeficientes de la variable (INDEPEN) son negativos y significativos, mientras que los coeficientes de la variable (DOMINI) son negativos, pero no significativos. Resultados que sugieren que el control ejercido por los consejeros independientes sobre el insider trading es superior al ejercido por los consejeros dominicales, y que están en línea con la hipótesis 3. 
TABLA 5

Efecto directo de la fracción de consejeros externos sobre el insider trading

\begin{tabular}{|c|c|c|c|c|}
\hline \multirow[b]{2}{*}{ Variables } & \multicolumn{2}{|c|}{ Panel A } & \multicolumn{2}{|c|}{ Panel B } \\
\hline & Coeff. & t-stat. & Coeff. & t-stat. \\
\hline Constante & 0,0056 & 0,309 & $-0,0184$ & $-1,125$ \\
\hline SH & $-0,0330^{* * *}$ & $-4,411$ & $-0,0346^{* * *}$ & $-4,595$ \\
\hline EXT & $-0,0247^{*}$ & $-1,771$ & $-0,0258^{*}$ & $-1,857$ \\
\hline$B S Z$ & $-0,0086^{*}$ & $-1,761$ & - & - \\
\hline$B S Z L^{1 /}$ & - & - & $0,0093^{*}$ & 1,909 \\
\hline$B S Z_{-} B^{2 /}$ & - & - & $-0,0051$ & $-1,294$ \\
\hline CONS & $-0,0016$ & $-0,532$ & $-0,0012$ & $-0,377$ \\
\hline$L V M K$ & $0,0056^{* * *}$ & 4,854 & $0,006^{* * *}$ & 5,054 \\
\hline$L E V$ & $-0,0117$ & $-1,623$ & $-0,011$ & $-1,524$ \\
\hline$B M$ & $0,0184^{* * * *}$ & 2,832 & $0,0193^{* * *}$ & 2,961 \\
\hline OPSZ & $0,0345^{* *}$ & 2,37 & $0,0347^{6 *}$ & 2,385 \\
\hline$O P M$ & 0,0015 & 0,447 & 0,0022 & 0,653 \\
\hline$R^{2}$ ajustado & \multicolumn{2}{|c|}{5,3} & \multicolumn{2}{|c|}{5,5} \\
\hline$F$ & \multicolumn{2}{|c|}{6,071} & \multicolumn{2}{|c|}{5,703} \\
\hline$N$ & \multicolumn{2}{|c|}{809} & \multicolumn{2}{|c|}{809} \\
\hline
\end{tabular}

Fuente: elaboración propia.

TABLA 6

Efecto directo del tipo de consejero sobre el insider trading

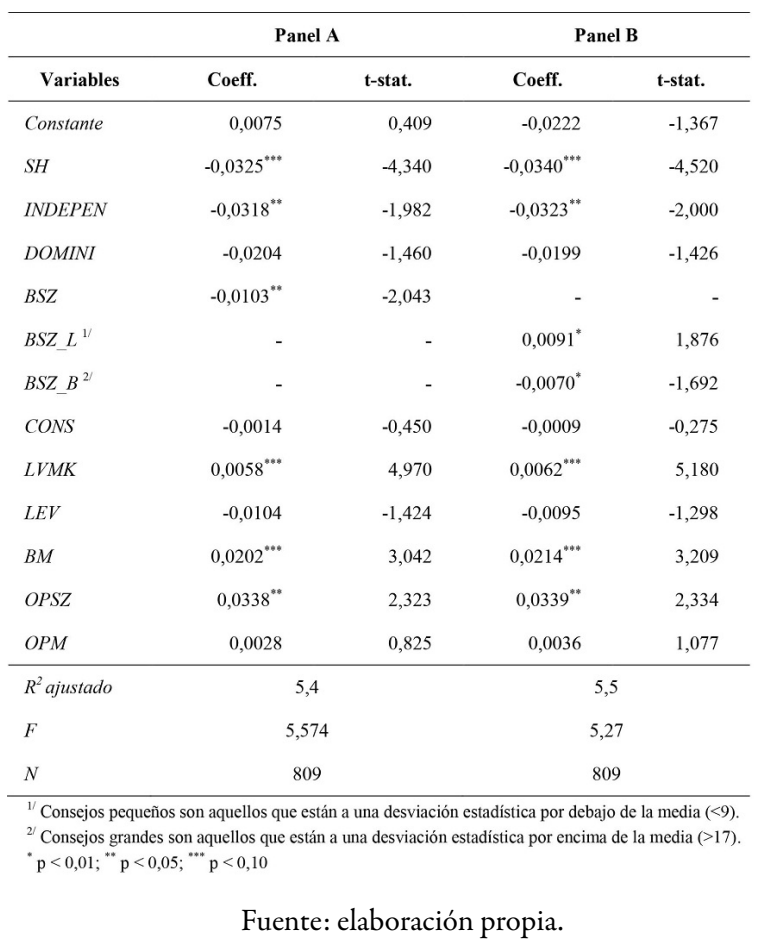

\section{Efecto moderador de la concentración de la propiedad sobre el rol de control de consejo sobre el insider trading}

Los resultados que permiten probar si la concentración de la propiedad modera el rol de control que realiza el consejo, en relación con el uso de información privilegiada por parte de los insiders, son recogidos en la tabla 7. El panel A muestra un coeficiente negativo, pero no significativo de la variable que representa el 
término de interacción entre la concentración de la propiedad y la fracción de consejeros externos $\left(S H^{*} E X T\right)$, como se planteó en la hipótesis 4, sugiriendo que la concentración de la propiedad disminuye el efecto disciplinante de los consejeros externos como grupo (evidenciado en la tabla 5). En el panel B de la tabla 7 se observa el mismo efecto moderador cuando se interactúa la concentración de propiedad y la fracción de consejeros independientes $\left(S H^{*} I N D E P\right)$. Por último, la interacción entre concentración de propiedad y la fracción de consejeros dominicales $\left(S H^{*} D O M I N I\right)$ muestra un coeficiente positivo y significativo, resultado que evidencia que la concentración de propiedad del principal accionista (alrededor de su media) modera negativamente el rol de control que ejercen los consejeros dominicales (panel C tabla 7); resultados que validan la hipótesis 5 , confirmando que los consejeros dominicales pueden estar más alineados con los intereses del gran accionista, en detrimento de los intereses de otros stakeholders.

TABLA 7

Efecto moderador de la concentración de la propiedad sobre el rol de control del consejo sobre el insider trading

\begin{tabular}{|c|c|c|c|c|c|c|}
\hline \multirow[b]{2}{*}{ Variables } & \multicolumn{2}{|c|}{ Panel A } & \multicolumn{2}{|c|}{ Panel B } & \multicolumn{2}{|c|}{ Panel C } \\
\hline & Coeff. & t-stat. & Coeff. & t-stat. & Coeff. & t-stat. \\
\hline Constante & $-0,0204$ & $-1,518$ & $-0,0211$ & $-1,579$ & $-0,0239^{*}$ & $-1,801$ \\
\hline$E X T$ & $-0,0022$ & $-1,421$ & $-0,0021$ & $-1,511$ & 0,0006 & 0,412 \\
\hline SH & $-0,0057^{s * 4}$ & $-4,168$ & $-0,0054^{s * x}$ & $-4,14$ & $-0,0050^{* * *}$ & $-3,758$ \\
\hline$S H^{*} E X T$ & $-0,0016$ & $-1,15$ & - & - & - & - \\
\hline INDEPEN & - & - & $-0,002$ & $-0,057$ & - & - \\
\hline$S H^{*} I N D E P$ & - & - & $-0,0004$ & $-0,697$ & - & - \\
\hline DOMINI & - & - & - & - & 0,001 & 0,412 \\
\hline$S H^{*} D O M I N I$ & - & - & - & - & $0,0023^{*}$ & 1,754 \\
\hline$B S Z$ & $-0,0093^{*}$ & $-1,903$ & $-0,0110^{* *}$ & $-2,175$ & $-0,0089^{*}$ & $-1,741$ \\
\hline CONS & $-0,0016$ & $-0,519$ & $-0,0004$ & $-0,133$ & 0 & $-0,015$ \\
\hline$L V M K$ & $0,0056^{* * 8}$ & 4,818 & $0,0058^{* * *}$ & 5,001 & $0,0057^{* *}$ & 4,867 \\
\hline$L E V$ & $-0,0105$ & $-1,442$ & $-0,0115$ & $-1,577$ & $-0,0128^{*}$ & $-1,763$ \\
\hline$B M$ & $0,0178^{* * *}$ & 2,731 & $0,0205^{* * *}$ & 3,086 & $0,0183^{* * *}$ & 2,753 \\
\hline OPSZ & $0,0341^{* *}$ & 2,347 & $0,0334^{* *}$ & 2,29 & $0,0337^{* *}$ & 2,312 \\
\hline$O P M$ & 0,0015 & 0,4607 & 0,0039 & 1,157 & 0,0043 & 1,239 \\
\hline$R^{2}$ ajustado & 5,4 & & 5,2 & & 5,3 & \\
\hline$F$ & 5,599 & & 5,398 & & 5,483 & \\
\hline$N$ & 809 & & 809 & & 809 & \\
\hline
\end{tabular}

\section{Variables de control}

Los resultados sugieren que algunas variables incluidas en los modelos afectan las rentabilidades anormales de las operaciones de compra de los insiders, lo cual valida la importancia de su control. Por ejemplo, existe una idea dominante en la literatura previa que sugiere que los problemas asociados a un gran consejo debilitan su función de control, existe literatura del insider trading que soportan esta idea validando una relación positiva entre el tamaño del consejo y la magnitud de las ganancias de las operaciones de los insiders (Ravina \& Sapienza, 2009; Rozanov, 2008).

Para el mercado español se encuentra una relación positiva y significativa entre el tamaño del consejo (BSZ) y el insider trading, lo que sugiere, que en consejos más grandes la información privilegiada es compartida con un mayor número de personas, lo que disminuye la oportunidad de explotar la ventaja informativa por parte de los insiders. Sin embargo, al separar los consejos entre grandes y pequeños se obtiene un coeficiente positivo y significativo de la variable ( $\left.B S Z \_L\right)$ que representa a los consejos muy pequeños, mientras que el coeficiente 
de la variable ( $\left.B S Z \_B\right)$ que representa los consejos muy grandes es negativo y significativo. Estos resultados indican que en empresas con consejos muy pequeños se presentan mayores oportunidades a los insiders de explotar su ventaja informativa, mientras que en empresas con consejos grandes estas ventajas disminuyen, lo cual puede atribuirse principalmente a la acelerada depreciación de la información al ser compartida por un número mayor de consejeros.

Se encontró una relación positiva entre el tamaño de la empresa y el insider trading. Sin embargo, vale apuntar que trabajos previos han arrojado resultados mixtos. Mientras que Seyhun (2000), Hillier \& Marshall (2002) y Fidrmuc et al. (2006) sustentan una relación negativa, Ke \& Petroni (2004) encuentran una relación positiva y Jeng et al. (2003) no soportan ninguna relación.

Este resultado sugiere que para el mercado español las grandes empresas ofrecen a los insiders mayores oportunidades de explotar su ventaja informativa en sus operaciones de compra. La relación positiva entre la ratio book to market está en línea con los resultados de trabajos previos sugiriendo que los insiders identifican empresas con altos ratios de book to market, y centran sus operaciones en este tipo de empresas (Friederich et al., 2002; Ikenberry, Lakonishok, \& Vermaelen, 1995). La relación negativa entre el nivel de endeudamiento de la empresa $(L E V)$ y el insider trading resalta la naturaleza disciplinante de la deuda ante el comportamiento oportunista de los insiders, resultados consistentes con el trabajo de Betzer \& Theissen (2009).

\section{Conclusiones}

En la última década ha surgido de nuevo el interés por analizar la eficacia de los sistemas de gobierno corporativo, fundamentalmente como consecuencia de escándalos financieros acaecidos a partir del año 2000 en grandes empresas de Estados Unidos y de Europa, que pusieron en entredicho el desarrollo de la gobernanza empresarial tal y como se había concebido hasta finales de la década de 1990, debido a su incapacidad para prevenir comportamientos oportunistas por parte de la dirección de la empresa. En este sentido, se planteó la necesidad de extender el análisis de la eficacia de los sistemas de gobierno corporativo, como mecanismos de control, a un terreno menos explorado y que, sin embargo, representa uno de los comportamientos oportunistas más detectados en la literatura financiera: el uso de información privilegiada por parte de los directivos empresariales, más conocido como insider trading.

Los resultados son concluyentes en relación con el papel de los mecanismos internos de control ya que ellos funcionan en línea con lo esperado desde la perspectiva de la teoría de la agencia. La relación negativa entre la concentración de la propiedad y el insider trading soporta la idea de que los grandes accionistas tienen el poder y los incentivos necesarios para controlar a los insiders. De igual manera, la relación negativa evidenciada entre la fracción de consejeros externos y el insider trading respalda el rol de control que se les presupone a estos consejeros. No obstante, se pudo comprobar que los consejeros independientes tienen un mejor desempeño en el control del insider trading, en relación con el control ejercido por los consejeros dominicales, resultados que están en línea con los planteamientos de la CNMV, en cuanto a la falta de independencia de estos consejeros.

En síntesis, los resultados permiten validar que tanto la concentración de la propiedad como la independencia del consejo tienen un efecto negativo sobre el insider trading cuando se analizan de manera independiente. Sin embargo, ese rol disciplinante de los consejos desaparece cuando se incorpora la concentración de propiedad como variable moderadora, haciéndose mucho más evidente en la interacción que se hace con la fracción de consejeros dominicales, donde se obtiene un coeficiente positivo y significativo.

\section{Consideraciones éticas}

La investigación no requirió aval ético. 


\section{Contribución de los autores}

Del Brío González realizó la coordinación general del artículo, Mora Valencia se dedicó a la revisión de la parte cuantitativa de la investigación y Tobar Arias se encargó del desarrollo general del manuscrito.

\section{Financiación}

El artículo contó con la financiación de la universidad ICESI a través del grupo de investigación: Inversión, Financiación y Control (código COL0012168).

\section{Conflictos de interés}

Los autores declaran que no existe conflicto de intereses asociado al desarrollo de la presente investigación y de la presentación y divulgación del manuscrito.

\section{Referencias}

Aboody, D., \& Lev, B. (2000). Information asymmetry, RyD, and insider gains. The Journal of Finance, 55, 2747-2766. https://doi.org/10.2307/222399

Agrawal, A., \& Knoeber, C. (1996). Firm performance and mechanisms to control agency problems between managers and shareholders. The Journal of Financial and Quantitative Analysis, 31(3), 377. https://doi.org/10.2307/23 31397

Agrawal, A., \& Mandelker, G. (1990). Large shareholders and the monitoring of managers: The case of antitakeover charter amendments. The Journal of Financial and Quantitative Analysis, 25(2), 143. https://doi.org/10.2307 $/ 2330821$

Aiken, L., West, S., \& Reno, R. (1991). Multiple regression: Testing and interpreting interactions. Sage Publications, Inc.

Alonso-Bonis, S., \& de Andrés-Alonso, P. (2007). Ownership structure and performance in large spanish companies. Empirical evidence in the context of an endogenous relation. Corporate Ownership and Control, 4(4 B), 206216. https://doi.org/10.22495/cocv4i4c1p5

Bathala, C., \& Rao, R. (1995). The determinants of board composition: An agency theory perspective. Managerial and Decision Economics, 16(1), 59-69. https://doi.org/10.1002/mde.4090160108

Beck, T., \& Levine, R. (2002). Industry growth and capital allocation: Does having a market- or bank-based system matter? Journal of Financial Economics, 64(2), 147-180. https://doi.org/10.1016/S0304-405X(02)00074-0

Berle, A., \& Means, G. (1932). The modern corporation and private property. New York: Macmillan Publishing Co.

Betzer, A., \& Theissen, E. (2009). Insider trading and corporate governance: The case of Germany. European Financial Management, 15(2), 402-429. https://doi.org/10.1111/j.1468-036X.2007.00422.x

Bhagat, S., \& Black, B. (2002). The non-correlation between board independence and long-term firm performance. Journal of Corporation Law, 27(2), 231-273. https://doi.org/10.2139/ssrn.133808

Bhattacharya, U., \& Daouk, H. (2002). The world price of insider trading. Journal of Finance, 57(1), 75-108. https: //doi.org/10.1111/1540-6261.00416

Bris, A. (2005). Do insider trading laws work? European Financial Management, 11(3), 267-312. https://doi.org/1 0.1111/j.1354-7798.2005.00285.x

Bushman, R. \& Smith, A. (2001). Financial accounting information and corporate governance. Journal of Accounting and Economics, 32(1-3), 237-333. https://doi.org/10.1016/S0165-4101(01)00027-1. 
Claessens, S., Djankov, S., Fan, J., \& Lang, L. (2002). Disentangling the incentive and entrenchment effects of large shareholdings. Journal of Finance, 57(6), 2741-2771. https://doi.org/10.1111/1540-6261.00511

Comisión Nacional del Mercado de Valores (CNMV). Código unificado de buen gobierno de las sociedades cotizadas (2006). Recuperado el 15 de junio de 2020 de https://www.cnmv.es/DocPortal/Publicaciones/CodigoGov/C odigo_unificado_Esp_04.pdf

Cziraki, P., De Goeij, P., \& Renneboog, L. (2014). Corporate governance rules and insider trading profits. Review of Finance, 18(1), 67-108. https://doi.org/10.1093/rof/rft001

Dah, M., Frye, M., \& Hurst, M. (2014). Board changes and CEO turnover: The unanticipated effects of the SarbanesOxley Act. Journal of Banking \& Finance, 41, 97-108. https://doi.org/10.1016/j.jbankfin.2014.01.006

De Jong, A., DeJong, D. V., Mertens, G., \& Wasley, C. E. (2005). The role of self-regulation in corporate governance: Evidence and implications from The Netherlands. Journal of Corporate Finance, 11(3), 473-503. https://doi.o $\mathrm{rg} / 10.1016 /$ j.jcorpfin.2004.01.002

Del Brío, E., De Miguel, A., \& Tobar, J. (2010). Efectos de la regulación bursátil sobre la eficiencia de los mercados de valores. Comparación entre España y Reino Unido. Revista Espanola de Financiacion y Contabilidad, 39(146), 321-342. https://www.jstor.org/stable/42782714

Del Brío, E., Miguel, A., \& Perote, J. (2002). An investigation of insider trading profits in the Spanish stock market. Quarterly Review of Economics and Finance, 42(1), 73-94. https://doi.org/10.1016/S1062-9769(01)00103-X

Del Brío, E., \& Perote, J. (2007). What enhances insider trading profitability? Atlantic Economic Journal, 35(2), $173-$ 188. https://doi.org/10.1007/s11293-006-9060-8

Demirgüç-Kunt, A., \& Maksimovic, V. (1998). Law, finance, and firm growth. Journal of Finance, 53(6), 2107-2137. https://doi.org/10.1111/0022-1082.00084

Demirgüç-Kunt, A., \& Maksimovic, V. (2002). Funding growth in bank-based and market-based financial systems: Evidence from firm-level data. Journal of Financial Economics, 65(3), 337-363. https://doi.org/10.1016/S030 4-405X(02)00145-9

Donnelly, R., \& Kelly, P. (2005). Ownership and board structures in Irish plcs. European Management Journal, 23(6), 730-740. https://doi.org/10.1016/j.emj.2005.10.016

Fama, E. F. (1980). Agency problems and the theory of the firm. Journal of Political Economy, 88(2), 288-307. http s://doi.org/10.1086/260866

Fama, E., \& Jensen, M. (1983). Separation of ownership and control. The Journal of Law y Economics, 26(2), 301-325. https://doi.org/10.2307/725104

Fidrmuc, J. P., Goergen, M., \& Renneboog, L. (2006). Insider trading, news releases, and ownership concentration. Journal of Finance, 61(6), 2931-2973. https://doi.org/10.1111/j.1540-6261.2006.01008.x

Fishman, M., \& Hagerty, K. (1992). Insider trading and the efficiency of stock prices. The RAND Journal of Economics, 23(1), 106. https://doi.org/10.2307/2555435

Friederich, S., Gregory, A., Matatko, J., \& Tonks, I. (2002). Short-run returns around the trades of corporate insiders on the London stock exchange. European Financial Management, 8(1), 7-30. https://doi.org/10.1111/1468036X.00174

Georgakopoulos, N. (1993). Insider trading as a transactional cost: A market micostructure justification and optimization of insider trading regulation. Connecticut Law Review, 26. https://heinonline.org/HOL/Page?ha ndle=hein.journals $/$ conlr26yid $=19 \mathrm{ydiv}=$ ycollection $=$

Goshen, Z., \& Parchomovsky, G. (2001). On insider trading, markets, and "negative" property rights in information. Virginia Law Review, 87(7), 1229. https://doi.org/10.2307/1073850

Grossman, S., \& Hart, O. (1986). The costs and benefits of ownership: A theory of vertical and lateral integration. Journal of Political Economy, 94(4), 691-719. https://doi.org/10.1086/261404

He, Q., \& Rui, O. (2016). Ownership Structure and Insider Trading: Evidence from China.Journal of Business Ethics, 134(4), 553-574. https://doi.org/10.1007/s10551-014-2384-4 
Hermalin, B., \& Weisbach, M. (1988). The determinants of board composition. The RAND Journal of Economics, 19(4), 589. https://doi.org/10.2307/2555459

Hillier, D., \& Marshall, A. (2002). Insider trading, tax-loss selling, and the turn-of-the-year effect. International Review of Financial Analysis, 11(1), 73-84. https://doi.org/10.1016/S1057-5219(01)00065-5

Howton, S. W. (2006). Effect of governance characteristics on the state of the firm after an initial public offering. Financial Review, 41(3), 419-433. https://doi.org/10.1111/j.1540-6288.2006.00150.x

Ikenberry, D., Lakonishok, J., \& Vermaelen, T. (1995). Market underreaction to open market share repurchases. Journal of Financial Economics, 39(2-3), 181-208. https://doi.org/10.1016/0304-405X(95)00826-Z

Jagolinzer, A. D. (2009). Sec Rule 10b5-1 and insiders' strategic trade. Management Science, 55(2), 224-239. https:/ /doi.org/10.1287/mnsc.1080.0928

Jeng, L. A., Metrick, A., \& Zeckhauser, R. (2003). Estimating the returns to insider trading: A performance-evaluation perspective. Review of Economics and Statistics, 85(2), 453-471. https://doi.org/10.1162/0034653037652999 36

Jensen, M. (1989). Eclipse of the Public Corporation. Managing the Multibusiness Company: Strategic Issues for Diversified Groups. https://books.google.com.co/books? $\mathrm{hl}=$ esylr=yid=_NIJtxxbgDMCyoi=fndypg=PA315ydq=Eclipse + of + the + Public

+Corporationyots=RA5TdeK0rOysig=7DTA06A3Wg_WoXhjiIyK_Rr5ocyredir_esc $=y \#$ \# $=$ onepageyq=Eclipse of the Public Corporationyf $=$ false

Jensen, M. C. (1993). The modern industrial revolution, exit, and the failure of internal control systems. The Journal of Finance, 48(3), 831-880. https://doi.org/10.1111/j.1540-6261.1993.tb04022.x

Jensen, M., \& Meckling, W. (1979). Theory of the firm: Managerial behavior, agency costs, and ownership structure BT. In K. Brunner (Ed.) Economics Social Institutions: Insights from the Conferences on Analysis and Ideology (pp. 163-231). Springer Netherlands. https://doi.org/10.1007/978-94-009-9257-3_8

Ke, B., y Petroni, K. (2004). How informed are actively trading institutional investors? Evidence from their trading behavior before a break in a string of consecutive earnings increases. Journal of Accounting Research, 42(5), 895927. https://doi.org/10.1111/j.1475-679X.2004.00160.x

Kim, K., Kitsabunnarat-Chatjuthamard, P., \& Nofsinger, J. (2007). Large shareholders, board independence, and minority shareholder rights: Evidence from Europe. Journal of Corporate Finance, 13(5), 859-880. https://doi .org/10.1016/j.jcorpfin.2007.09.001

Klein, A. (1998). Firm performance and board committee structure. Journal of Law and Economics, 41(1), 275-303. https://doi.org/10.1086/467391

Korczak, A., Korczak, P., \& Lasfer, M. (2010). To trade or not to trade: The strategic trading of insiders around news announcements. Journal of Business Finance and Accounting, 37(3-4), 369-407. https://doi.org/10.1111/j.14 68-5957.2010.02185.x

La Porta, R., Lopez-de-Silanes, F., \& Shleifer, A. (1999). Corporate ownership around the world. Journal of Finance, 54(2), 471-517. https://doi.org/10.1111/0022-1082.00115

La Porta, R., Lopez-de-Silanes, F., Shleifer, A., \& Vishny, R. W. (1998). Law and finance. Journal of Political Economy, 106(6), 1113-1155. https://doi.org/10.1086/250042

La Porta, R., Lopez-De-Silanes, F., Shleifer, A., \& Vishny, R. W. (1997). Legal determinants of external finance.Journal of Finance, 52(3), 1131-1150. https://doi.org/10.1111/j.1540-6261.1997.tb02727.x

Lakonishok, J., \& Lee, I. (2001). Are Insider Trades Informative? The Review of Financial Studies, 14(1), 79-111. ht tp://www.jstor.org/stable/2696757

Lin, C., Ma, Y., \& Su, D. (2009). Corporate governance and firm efficiency: Evidence from China's publicly listed firms. Managerial and Decision Economics, 30(3), 193-209. https://doi.org/10.1002/mde.1447

Maug, E. (1998). Large shareholders as monitors: Is there a trade-off between liquidity and control? Journal of Finance, 53(1), 65-98. https://doi.org/10.1111/0022-1082.35053 
Méndez, C., \& García, R. (2007). The effects of ownership structure and board composition on the audit committee meeting frequency: Spanish evidence. In Corporate Governance: An International Review (Vol. 15, pp. 909-922). Blackwell Publishing Ltd. https://doi.org/10.1111/j.1467-8683.2007.00619.x

Morck, R., Shleifer, A., \& Vishny, R. W. (1988). Management ownership and market valuation. An empirical analysis. Journal of Financial Economics, 20(C), 293-315. https://doi.org/10.1016/0304-405X(88)90048-7

Park, Y. W., \& Shin, H. H. (2004). Board composition and earnings management in Canada. Journal of Corporate Finance, 10(3), 431-457. https://doi.org/10.1016/S0929-1199(03)00025-7

Qiu, Y., He, H., \& Xiao, G. (2018). The information content of insider trading: Evidence from China. Finance Research Letters, 26, 126-131. https://doi.org/10.1016/j.frl.2017.12.007

Rajan, R., \&Zingales, L. (1996). FinancialDependence and Growth. Cambridge, MA. https://doi.org/10.3386/w5758

Ravina, E., \& Sapienza, P. (2009). What Do Independent Directors Know? Evidence from Their Trading. Review of Financial Studies, 23(3), 962-1003. https://doi.org/10.1093/rfs/hhp027

Rogoff, D. (1964). the forecasting properties of insiders' transactions. The Journal of Finance, 19(4), 697. https://do i.org/10.2307/2977128

Romano, R. (1998). Empowering investors: A market approach to securities regulation. Yale Law Journal, 107(8), 2359-2427. https://doi.org/10.2307/797346.

Rozanov, K. (2008). Corporate governance and insider trading. Recuperado el 30 de septiembre de 2021 de https://d space.mit.edu/bitstream/handle/1721.1/45333/315871878-MIT.pdf?sequence $=2$.

Seyhun, H. N. (2000). Investment intelligence from insider trading. MIT Press.

Shleifer, A., \& Vishny, R. (1986). Large shareholders and corporate control. Journal of Political Economy, 94(3, Part 1), 461-488. https://doi.org/10.1086/261385

Shleifer, A., \& Vishny, R. (1997). The limits of arbitrage. Journal of Finance, 52(1), 35-55. https://doi.org/10.1111 /j.1540-6261.1997.tb03807.x

Tobar, J. E., Del Brío, E. B., \& De Miguel, A. (2017). El efecto de los mecanismos internos de control en las operacionescon información privilegiada. Estudios Gerenciales, 33 (228-239). https://doi.org/10.1016/j.estger .2017 .07 .002

Yermack, D. (1996). Higher market valuation of companies with a small board of directors. Journal of Financial Economics, 40(2), 185-211. https://doi.org/10.1016/0304-405X(95)00844-5

Zhou, X. (2001). Understanding the determinants of managerial ownership and the link between ownership and performance: Comment. Journal of Financial Economics, 62(3), 559-571. https://doi.org/10.1016/S0304-405 $\mathrm{X}(01) 00085-\mathrm{X}$

\section{Notas}

* Artículo de investigación

1 La principal justificación para la regulación en contra del insider trading es el problema de selección adversa (modelo Akerlof de 1970) que le causa a los agentes menos informados.

2 Agradecemos la sugerencia del revisor anónimo sobre la necesidad y la forma de sustentar la relación causal entre las variables de gobierno corporativo y el insider trading.

3 Este trabajo tratará de forma indistinta rentabilidades anormales o insider trading.

4 Los consejeros externos independientes no tienen vínculos con la propiedad de la empresa y se les designa por su experiencia y reputación profesional, representan los intereses de los pequeños accionistas. Los consejeros externos dominicales sí tienen vínculos profesionales o personales con la propiedad de la empresa, a pesar de no formar parte del equipo directivo, representan los intereses de los grupos accionariales de referencia.

5 Por ejemplo, para España en el código unificado de buen gobierno recomienda que los consejeros externos sean mayoría en el consejo de administración (recomendación 10), que el número de consejeros independientes represente, al menos un tercio del total de consejeros (recomendación 13). Sugiere también que las diferentes comisiones estén preferiblemente compuestas por consejeros externos y presididas por un consejero independiente (recomendación 44) (CNMV, 2006). Los principios de gobierno corporativo divulgados por la OCDE confieren especial relevancia a los 
consejeros independientes, especialmente a la hora de emitir juicios objetivos Literal "E", p. 57, responsabilidades del consejo.

6 Los autores utilizan variables dummy que identifican el tipo de accionista cuando estos tienen una participación al menos del $5 \%$ del capital.

7 Código unificado de buen gobierno para las empresas cotizadas (CNMV, 2006).

8 La regulación española considera la posesión de un porcentaje significativo de acciones o su representación como una causa de pérdida de independencia por parte de los consejeros dominicales (un consejero deja de ser independiente si su participación en el capital de la empresa supera el 5\%) (CNMV, 2006).

\section{Licencia Creative Commons CC BY 4.0}

Para citar este artículo: Tobar, J., Mora, A., \& Del Brío, E. (2021). Gobierno corporativo e insider trading: la concentración de la propiedad como variable moderadora del rol de control de los consejos. Cuadernos de Administración, 34. https://doi.org/10.11144/Javeriana.cao34.gcitcp 